\title{
PROMOÇÃO DE ISOLAMENTO SOCIAL NA PANDEMIA DE COVID-19: CONSIDERAÇÕES DA ANÁLISE COMPORTAMENTAL DA CULTURA
}

\author{
PROMOTING SOCIAL ISOLATION IN THE COVID-19 PANDEMIC: CONSIDERATIONS OF THE \\ BEHAVIORAL ANALYSIS OF CULTURE
}

\author{
VIRGÍNIA CORDEIRO AMORIM12 - ORCID 0000-0001-8827-4230 \\ THAIS MARIA MONTEIRO GUIMARÃES ${ }^{23}$ - ORCID 0000-0003-3938-3937 \\ JOÃO ARISTIDES TOMAZ DE ALMEIDA ${ }^{2}$ - ORCID 0000-0001-7939-8813 \\ YAN VALDERLON² - ORCID 0000-0003-1801-1069 \\ MIGUEL ABDALA ${ }^{4}$ - ORCID 0000-0002-7842-4191 \\ 1 UNIVERSIDADE FEDERAL DE MATO GROSSO, BRASIL \\ 2 UNIVERSIDADE FEDERAL DO PARÁ, BRASIL \\ ${ }^{3}$ BEEHAVE: SOLUÇÕES COMPORTAMENTAIS, BRASIL \\ ${ }^{4}$ UNIVERSIDADE FEDERAL DO CEARÁ, BRASIL
}

\begin{abstract}
RESUMO
O mundo enfrenta atualmente a pandemia de COVID-19 (Corona Virus Disease, Doença do Coronavírus). Como até o momento não há vacina disponível, estudos recentes apontam estratégias não farmacêuticas como as mais eficazes para a evitação de novos contágios, como o isolamento social. Diante disso, é necessário compreender os procedimentos culturais/comportamentais e as variáveis envolvidas na promoção do isolamento social para controlar a COVID-19. Assim sendo, são objetivos deste artigo: a) descrever metacontingências e macrocontingências envolvidas nas estratégias adotadas para promoção do isolamento social e b) discutir tais medidas como possíveis estratégias de planejamento cultural. Para tal, consultamos estratégias de combate à pandemia adotadas pelo Governo do Estado de São Paulo. Foram encontradas ações governamentais que podem ser descritas como metacontingências com objetivo de promover macrocomportamentos e entrelaçamentos de contingências que aumentem o Índice de Isolamento Social. Tal índice, por sua vez, afetaria a taxa de contágios por COVID-19. Discutimos os impactos das estratégias de combate à pandemia à luz do planejamento cultural. Esperamos que as discussões abordadas no presente trabalho possam auxiliar no planejamento de estratégias para promoção de isolamento social e da consequente sobrevivência das culturas.
\end{abstract}

Palavras-chave: COVID-19; Isolamento Social; Metacontingência; Macrocontingência; Planejamento Cultural.

\begin{abstract}
The world is currently facing the COVID-19 pandemic (Corona Virus Disease). As there is no vaccine available so far, recent studies have pointed out non-pharmaceutical strategies as the most effective in preventing new infections, such as social isolation. Therefore, it is necessary to understand cultural/behavioral procedures and variables involved in promoting social isolation to control COVID-19. Thus, the objectives of this article are: a) to describe metacontingencies and macrocontingencies involved in the strategies adopted to promote social isolation and $b$ ) to discuss such measures as possible cultural design strategies. To this end, we consulted strategies to combat the pandemic adopted by the Government of the State of São Paulo. Government actions were found that can be described as metacontingencies, with the objective of promoting macrobehaviors and interlocking contingencies that increase the Social Isolation Index. Such index, in turn, would affect the rate of contagion by COVID-19. We discussed impacts of strategies to combat the pandemic in the light of cultural design. We hope that discussions covered in this work can help in planning strategies to promote social isolation, and consequent survival of cultures.
\end{abstract}

Keywords: COVID-19; Social isolation; Metacontingency; Macrocontingency; Cultural Design.

\footnotetext{
O presente trabalho foi financiado parcialmente através de bolsas de doutorado do $3^{\circ}$ e $4^{\circ}$ autores pelo Conselho Nacional de Desenvolvimento Científico e Tecnológico (CNPq) e pela Coordenação de Aperfeiçoamento de Pessoal de Nível Superior (CAPES)), respectivamente. Agradecemosaos profissionais de saúde e de serviços essenciais pelo enfrentamento da pandemia de COVID-19, aos cientistas que produzem conhecimentos e tecnologias relacionados a essa doença, à REBAC pela convocação aos analistas do comportamento a produzirem conhecimento sobre o comportamento humano em épocas como essa de pandemia e aos revisores pelas sugestões e comentários sobre este trabalho. Correspondências sobre este artigo devem ser endereçadas a: virginiacordeiroamorim@gmail.com
}

DOI: http://dx.doi.org/10.18542/rebac.v16i1.8886 
O mundo enfrenta a pandemia de COVID-19 (Corona Virus Disease, Doença do Coronavírus), doença respiratória contagiosa causada pelo Sars-Cov-2 (Severe acute respiratory syndrome coronavirus 2, Coronavírus da síndrome respiratória aguda grave 2). Singhal (2020) descreveu os sintomas da COVID-19 como semelhantes a uma gripe comum (tosse, febre, dor de garganta, fadiga, dor de cabeça e falta de ar), mas com um alto poder de contágio, principalmente, por meio do contato com gotículas infectadas.

Diante do aumento expressivo de casos de COVID-19, a Organização Mundial da Saúde (OMS) declarou a contaminação pelo novo coronavírus como uma emergência de saúde pública de importância internacional, sendo decretada como pandemia no dia 11 de março de 2020 (OPAS/OMS Brasil, 2020). Segundo a OMS (World Health Organization, 2020), no mundo, até o dia 22 de junho de 2020, já tinham sido registrados quase 9 milhões de casos confirmados com mais de 465 mil mortes. No Brasil já foram registrados mais de 1 milhão de casos confirmados com quase 50 mil mortes.

Como não há, até o momento, uma vacina contra a COVID-19, intervenções não farmacêuticas (e.g., rastreamento de contatos a partir da pessoa infectada, quarentena e distanciamento social) são os únicos meios imediatos para reduzir a transmissão de Sars-CoV-2 (Anderson, Heesterbeek, Klinkenberg, \& Hollingsworth, 2020; Ferguson et al., 2020; Kissler, Tedijanto, Goldstein, Grad, \& Lipsitch, 2020). O estudo de Walker et al. (2020) simula a adoção de diferentes alternativas para conter a transmissão do vírus, estimando que, caso medidas de mitigação não fossem adotadas, haveria bilhões de pessoas infectadas e milhões de mortes no mundo todo. No Brasil, a recomendação geral tem sido de que, exceto trabalhadores de setores essenciais (e.g., saúde), todos permaneçam em suas casas e saiam apenas em caso de extrema necessidade, resguardando dois metros de distância de outras pessoas nessas ocasiões. Apesar de tal recomendação poder ser definida tecnicamente como distanciamento social, a sua eficácia vem sendo noticiada e avaliada por órgãos oficiais através de um indicador de “isolamento social” (Governo de São Paulo, 2020a), central às análises do presente trabalho. Por esse motivo, utilizaremos o termo isolamento social daqui em diante.

Talvez demore até que uma vacina eficaz esteja disponível em escala global (Kissler et al., 2020). Isto posto, estudos que demonstrem os meios pelos quais intervenções não farmacêuticas ocorrem são imprescindíveis. Tais estudos podem evidenciar tanto variáveis que estão sendo manipuladas ou a serem manipuladas nos próximos períodos de isolamento social para controlar a COVID-19, quanto os procedimentos culturais e comportamentais envolvidos nas estratégias de intervenção em larga escala que sirvam de base para projetos de planejamento cultural de uma forma mais ampla.

Nesse sentido, algumas pesquisas da Análise Comportamental da Cultura têm investigado fenômenos sociais a partir de duas unidades de análise, metacontingência e macrocontingência, as quais podem ser úteis no cenário atual de pandemia. Metacontingência se refere à relação funcional entre culturantes (contingências comportamentais entrelaçadas - CCEs - e seus produtos agregados - PAs) e consequências culturais (Glenn, 2003; Glenn et al., 2016). Macrocontingência se refere à relação de comportamentos operantes e/ou CCEs com um efeito cumulativo de relevância social (Glenn et al., 2016). Na macrocontingência, a soma dos resultados de respostas de indivíduos mantidos por consequências operantes (em alguns casos, somados aos PAs de metacontingências) podem produzir efeitos ambientais que resultam em problemas sociais. Esses comportamentos operantes, aprendidos socialmente e recorrentes entre membros de um sistema cultural, são denominados de macrocomportamentos (Glenn et al., 2016).

Apesar da baixa produção de pesquisas aplicadas envolvendo a metacontingência (Zilio, 2019), o uso dessa unidade de análise em estudos interpretativos, especialmente naqueles que avaliaram os procedimentos adotados (e.g., Bortoloti \& D’Agostino, 2007; Lé SénéchalMachado \& Todorov, 2008) e efeitos (e.g., Fava, 2014) de intervenções em larga escala, assim como seu uso no delineamento de uma intervenção para modificar práticas culturais (Lemos, 2018), sugere que essa unidade de análise seja profícua para intervenções culturais numa perspectiva de planejamento cultural (Valderlon \& Elias, 2019). Macrocontingência também tem sido uma unidade de análise utilizada em pesquisas interpretativas para descrever e discutir práticas culturais estabelecidas em larga escala, como corrupção, desastres climáticos, leis da saúde, sistema público de educação, desperdício de alimentos (e.g., Agbota, Sandaker, de Carvalho, \& Couto, 2017; Ardila Sánchez, Houmanfar, \& Alavosius, 2019; da Hora \& Sampaio, 2019; Martins, 2009; Ellis \& Magee, 2007; Tagliabue \& Sandaker, 2019). Apesar de alguns estudos ressaltarem uma das unidades de análise, grande parte deles se utiliza de ambas em suas interpretações. Parece-nos, então, que compreender um fenômeno cultural de tamanha complexidade, como a participação do comportamento das pessoas na propagação de uma pandemia, demanda uma análise que contemple as duas unidades descritas.

Assim sendo, são objetivos deste artigo, considerando uma localidade brasileira: a) descrever metacontingências e macrocontingências envolvidas nas medidas adotadas para promoção do isolamento social e b) discutir tais medidas como possíveis estratégias de planejamento cultural.

No contexto brasileiro, deliberações sobre a implementação das recomendações da OMS para o isolamento social são tomadas pelo Governo Federal/Ministério da Saúde. Entretanto, elas estão sujeitas a serem ou não adotadas pelos governos estaduais (Ajzenman, Cavalcanti, \& Da Mata, 2020; Correio Braziliense, 2020). Além disso, Ajzenman et al. (2020) verificaram que, após o Presidente Jair Bolsonaro ter descartado publicamente os riscos associados à pandemia e ter desaconselhado as medidas de isolamento social, a adesão ao isolamento foi menor em localidades onde predomina uma postura a favor do Presidente do que naquelas onde ele tem menos apoio. Essas constatações sugerem que a análise em um âmbito mais 
restrito tende a ser mais acurada, ainda que sujeita aos efeitos de decisões federais.

Até o dia 25 de abril de 2020, momento em que os registros do Painel Coronavírus (Ministério da Saúde, 2020) foram consultados, os cinco estados brasileiros mais afetados, isto é, com maior taxa de letalidade (Número de óbitos por COVID-19 em determinada área e período multiplicado por 100 ou 1000, dividido pelo número total de pessoas com a doença na mesma área e período), foram, em ordem decrescente, Paraíba, Rio de Janeiro, São Paulo, Pernambuco e Amazonas. Sabemos, entretanto, que entre os países mais afetados pela COVID-19, o Brasil é o que menos faz testes para detectar a doença na população, implicando em uma reconhecida subnotificação (Azevedo, 2020). Considerando que o achatamento da curva de contágio pela doença demanda adoção de medidas que visam o índice de isolamento social (IIS), entendemos que esse consiste em um indicador relevante a respeito da efetividade das medidas. Logo, os sites oficiais desses cinco governos estaduais foram consultados em busca de informações sobre: a) o estabelecimento de um IIS ideal, b) o monitoramento dos IISs durante a quarentena e c) as medidas adotadas para alcançar o IIS ideal. Dentre os cinco estados, São Paulo (Governo de São Paulo, 2020b) foi o que apresentou informações com maiores detalhes e clareza sobre esses três aspectos em análise.

Visto que lidar com informações sobre o monitoramento do IIS e a implantação de medidas de controle da COVID-19 em nível municipal daria mais precisão à análise, foi feita uma consulta ao site oficial do Governo da Cidade de São Paulo (Cidade de São Paulo, 2020). Entretanto, até o momento, as informações buscadas foram apresentadas com mais clareza pela esfera estadual. Em vista da descentralização para a tomada de decisão em relação às recomendações do Ministério da Saúde e do Governo Federal e de como as informações estão organizadas, o presente estudo terá como foco de análise as medidas propostas pelo governo do estado de São Paulo.

\section{ANÁLISE DE ESTRATÉGIAS IMPLEMENTADAS PELO ESTADO DE SÃO PAULO PARA A PROMOÇÃO DE ISOLAMENTO SOCIAL.}

Quanto ao estabelecimento de um IIS ideal, o Centro de Contingência do Coronavírus da Secretaria de Estado da Saúde - SP preconiza, até o momento, a adesão de $70 \%$ da população para que a disseminação de COVID-19 seja controlada no estado (Secretaria de Estado da Saúde, 2020). Possivelmente essa porcentagem é resultado do número de reprodução básica (i.e., número geral de pessoas que provavelmente seriam infectadas sem medidas de mitigação) da China, que foi cerca de $60 \%$ no estágio inicial da epidemia, indicando, assim, que $70 \%$ deve ser o IIS para que haja controle eficiente da transmissão da COVID-19 (Anderson et al., 2020).

Sobre o monitoramento do IIS, o Governo de São Paulo implantou o Sistema de Monitoramento Inteligente de São Paulo - Simi-SP (Governo de São Paulo, 2020c), o qual informa o IIS por cidade, desde 05 de maio de 2020, e envia mensagens de alerta para regiões com maior incidência da doença. Esse monitoramento se fez possível através de uma parceria do estado com operadoras de telefonia celular, o que permite que o Simi-SP acesse informações georreferenciadas de mobilidade urbana em tempo real, partindo de dados coletivos de aglomerados a partir de 30 mil pessoas. Relatórios diários apontam as cidades e regiões com maior ou menor adesão ao isolamento e promovem campanhas de conscientização (Governo de São Paulo, 2020d). Segundo o Simi-SP, esse monitoramento só ocorrerá durante a pandemia e análises de dados individuais nunca são conduzidas, respeitando a privacidade dos usuários. No presente trabalho, usamos o IIS como um indicador relevante das medidas de controle da disseminação do coronavírus. A discussão acerca das implicações éticas dos procedimentos adotados para obtêlo, assim como a verificação da correspondência entre as declarações do governo e as ações realmente conduzidas são objetos de estudos relevantes para os analistas do comportamento, mas fogem ao escopo do presente trabalho.

Por último, o governo estadual mantém atualizada em seu site (Governo de São Paulo, 2020b) uma página com informações sobre as medidas tomadas em nove áreas: 1) Saúde, 2) Medidas Administrativas, 3) Restrição de Circulação, 4) Servidores e Máquina Pública, 5) Social, 6) Educação, 7) Incentivo à economia, 8) Comunicação e 9) Outros agentes e Poderes. Como o presente artigo aborda o isolamento social, as medidas tratadas como promotoras de Restrição de Circulação serão o foco prioritário da análise.

A Figura 1 mostra o encadeamento de eventos que antecederam e que sucederam as prescrições do Governo de SP sobre as medidas de Restrição de Circulação. Podemos dizer que a pandemia de COVID-19, juntamente com os primeiros casos de contaminação no Brasil, o grande número de mortos em outros países, as fragilidades do nosso sistema de saúde, as estimativas de danos, entre outros eventos, consistiram em condições antecedentes (Vieira, Andery, \& Pessôa, 2016) para CCEs ocorridas no Governo de SP que tiveram as prescrições de medidas como PAs. Como a implementação de tais medidas envolvem vários setores (escolas estaduais, órgãos públicos, comércio etc.), supomos que as deliberações envolvidas na prescrição das medidas e no estabelecimento das datas em que passariam a vigorar tenham envolvido entrelaçamentos de inúmeros agentes e órgãos. Possivelmente, ocorreram várias metacontingências envolvendo alguns agentes/órgãos específicos, em momentos distintos do enfrentamento à pandemia, que culminaram em cada uma das prescrições como PA proveniente das CCEs do Governo de SP.

Os cinco retângulos que mostram os PAs foram organizados de forma que: a) acima de cada um deles, sobre um fundo preto, estão descritos os tipos de medidas prescritas e b) dentro dos retângulos encontram-se os serviços/órgãos/práticas afetados, seguidos das datas em que a medida entrou em vigor. No retângulo superior encontramse órgãos/espaços/eventos sobre os quais o estado tem ingerência direta. Portanto, determinações, fechamentos e suspensões foram prescritos logo no começo da pandemia no Brasil. $O$ segundo retângulo apresenta órgãos/espaços/eventos de cunho privado, sobre os quais o estado tem menor ingerência. Dessa forma, as medidas de fechamento/suspensão tiveram caráter de recomendação. 


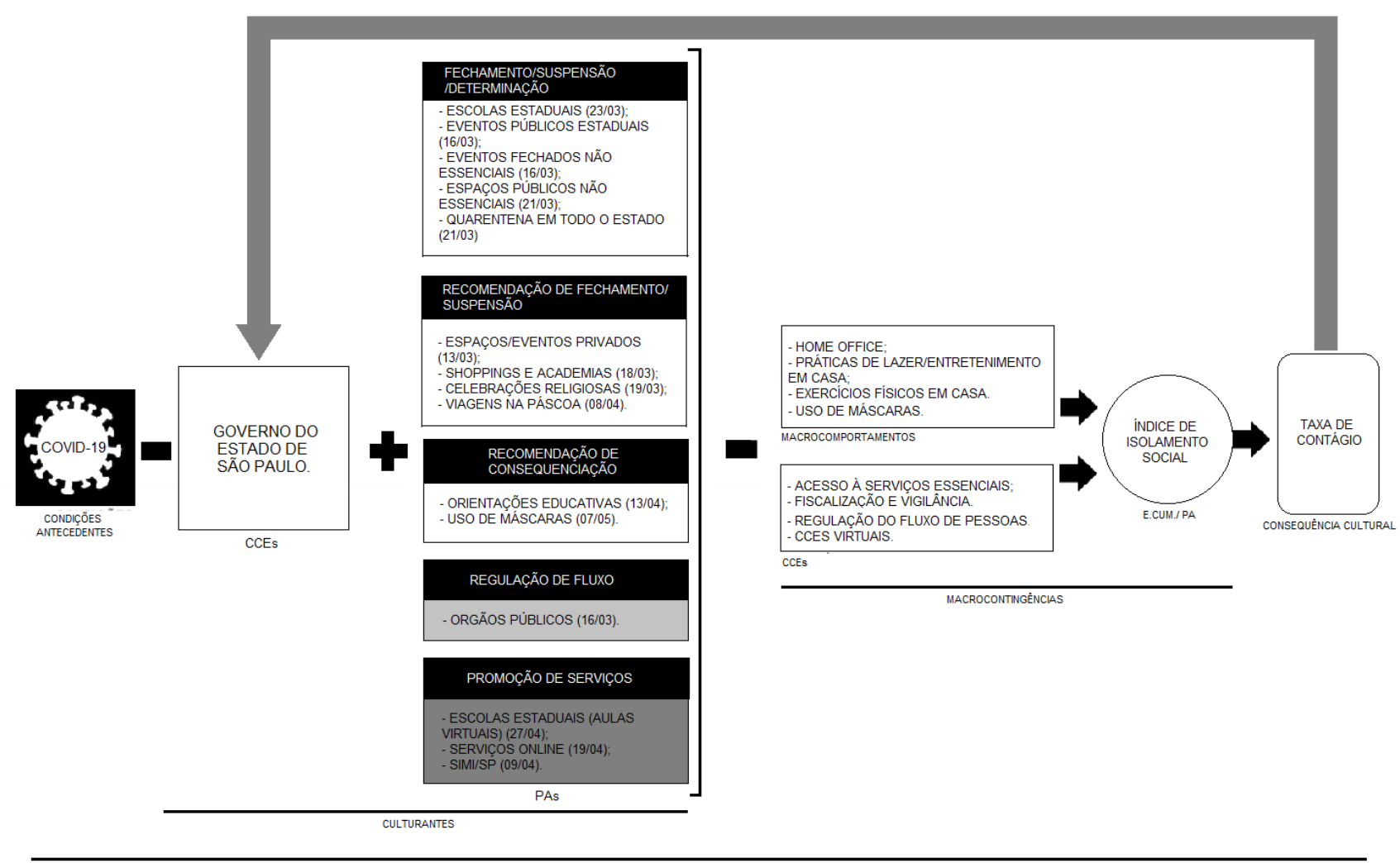

METACONTINGÊNCIA

Figura 1. Metacontingência do Governo de SP para promoção de Isolamento Social. CCEs = Contingências Comportamentais Entrelaçadas; PAs = Produtos Agregados; E.CUM/PA = Efeito Cumulativo ou Produto Agregado.

No terceiro retângulo, observamos que, poucas semanas após o começo dessas recomendações e do estabelecimento da quarentena (21 de março de 2020), o estado recomendou Orientações Educativas (13 de abril de 2020) a comércios e serviços que descumprissem a quarentena. Centenas de agentes (funcionários do estado, Polícia Militar e Vigilância Sanitária) passaram a agir coordenadamente para monitorar, fiscalizar e, em um primeiro momento, advertir os responsáveis pelos estabelecimentos sobre a obrigatoriedade de seguir a recomendação. Caso não atendam voluntariamente à recomendação, técnicos de saúde estadual, acompanhados de policiais militares, devem notificar os estabelecimentos e proceder à sua interdição (Governo de São Paulo, 2020d). O uso de máscaras, medida cuja adoção em espaços públicos foi recomendada em 23 de abril de 2020 , tornou-se obrigatório em transportes públicos em 29 de abril de 2020 e para qualquer atividade em espaços públicos em 07 de maio de 2020. No dia 04 de maio de 2020, o governo estadual delegou às prefeituras a responsabilidade pela regulamentação dessa medida, fiscalização e aplicação de penalidades (Governo de São Paulo, 2020e).

Observamos que as medidas prescritas nos três primeiros retângulos têm como foco a diminuição da frequência de CCEs que já ocorriam antes da pandemia e envolviam contato social em massa (e.g., funcionamento de escolas) com grande potencial de disseminação da
COVID-19. Destacamos, contudo, que há diferenças funcionais entre tais medidas. As prescrições agrupadas nos dois retângulos superiores constituem PAs de CCEs do Governo que provavelmente adquirem também a função de estímulo discriminativo para operantes (apresentados nos macrocomportamentos da Figura 1). Já o PA denominado Orientações Educativas, constitui uma recomendação para consequenciação de comportamentos e CCEs que venham a ocorrer.

Apesar de enfocar a supressão de CCEs entre os membros da população de uma forma geral, a implantação das medidas de isolamento demanda necessariamente a promoção de novas CCEs dos agentes públicos por elas responsáveis. Então, esse é um PA que se converte em condição antecedente para novas CCEs envolvidas na implantação da medida (apresentadas no retângulo abaixo dos macrocomportamentos na Figura 1). Como a medida anuncia advertência, notificação e interdição, também pode ter função de estímulo discriminativo em contingências de esquiva para a população. Funções semelhantes podem ser exercidas pela prescrição do uso de máscaras, que além de produto agregado, se converte em condição antecedente para CCEs necessárias à fiscalização e penalização na esfera municipal e como estímulo discriminativo para o uso de máscaras.

O quarto retângulo (em cinza claro), representando os PAs do governo, mostra Órgãos Públicos sobre os quais o estado também intervém diretamente. 
Mas, por prestarem serviços indispensáveis (Poupatempo, Restaurantes Bom Prato, Detran, Centro de Integração e Cidadania), o estado prescreveu restrições de acesso, regulando o fluxo de entrada nestas repartições públicas. Isto é, observamos prescrições para diminuir e alterar, mas não de suprimir completamente as interações presenciais. Adicionalmente, esse PA deve ter função de estímulo discriminativo para macrocomportamentos de evitação destes estabelecimentos, além de alterar as CCEs entre os funcionários e a população para regular o fluxo de pessoas (e.g., aferição de temperatura, fiscalização do distanciamento das pessoas ao entrarem nesses estabelecimentos).

As prescrições de medidas do último retângulo (em cinza escuro), diferentemente das anteriores, constituem PAs que servem de condições antecedentes para a ocorrência de novas interações (CCEs envolvidas na execução de aulas virtuais, no aumento de prestação de serviços on-line e nas atividades do Simi-SP), as quais devem ocorrer essencialmente através da internet e de georreferenciamento. Aqui destacamos que a promoção do aumento do IIS (que implica em diminuição de CCEs presenciais), tem como suporte a promoção de novos tipos de CCEs, essencialmente virtuais, e que os PAs são prescrições que também funcionam como antecedentes para os macrocomportamentos e as novas CCEs envolvidos no seu cumprimento e implantação.

Sobre as macrocontingências envolvidas na promoção do isolamento social, espera-se que tanto a promoção de macrocomportamentos (e.g., permanência dos indivíduos em suas residências), quanto os PAs das novas CCEs ocasionadas pelas medidas governamentais (e.g., fiscalização e vigilância) favoreçam o aumento do IIS.

Nesse sentido, alguns macrocomportamentos devem ser almejados. Os exemplos são descritos no retângulo "macrocomportamentos", da Figura 1. O home office permite que atividades laborais, tradicionalmente externas, ocorram em domicílio, contribuindo com o PA de promoção de serviços online. Essas respostas se mantêm pelo salário e permanência no emprego (consequências individuais) durante o período de quarentena. Outro exemplo são as práticas de lazer e entretenimento dentro de casa, como assistir a shows e filmes pela internet, mantidas por consequências reforçadoras intrínsecas. Por fim, podemos citar o exercitar-se na própria residência, visto que esportes costumam ser praticados em locais com grupos de pessoas. Essas respostas, agora ocorrendo em ambiente isolado, são mantidas por reforçadores naturais e arbitrários individuais.

Além dos macrocomportamentos relativos à permanência dos indivíduos em suas residências, outras respostas mantidas por consequências individuais podem ser emitidas, como o uso de máscaras de proteção e a higienização das mãos em locais públicos. A topografia de respostas sociais também é alterada em isolamento, visto que para um indivíduo interagir com outro é necessária a mediação de um sistema de interação à distância, como redes sociais. Dessa maneira, utilizar essas tecnologias para comunicação também é um operante que cumpre as normas de isolamento social.

Outros entrelaçamentos (ver retângulo "CCEs" abaixo de macrocomportamentos da Figura 1), produtos dos culturantes da regulação de fluxo em órgãos públicos (e.g., acesso aos diversos serviços essenciais, que permanecem em funcionamento, tomando medidas necessárias) podem ser analisados como associados aos macrocomportamentos gerando o efeito cumulativo de aumento do IIS. Para a resposta de comprar suprimentos de alimentação e remédios, torna-se necessária a interação social com algum funcionário desses estabelecimentos, o qual assume a função de contexto. Essa relação mantida pela aquisição do produto, porém qualquer contato físico é evitado com o uso dos equipamentos de proteção individuais (e.g., máscaras, luvas). Em agências bancárias, o contato social respeita as mesmas regras, com o adicional da regulação do distanciamento de dois metros entre pessoas nas filas. Por fim, agentes da prefeitura também são instruídos a orientar pessoas aglomeradas em locais públicos, sendo mais um exemplo de entrelaçamentos relacionados com o aumento do IIS.

Como visto na Figura 1, a soma destes macrocomportamentos e das novas CCEs resultam em um IIS, descrito como efeito cumulativo. Esse índice não constitui, por si só, um evento capaz de selecionar os operantes e CCEs anteriormente descritos. Ou seja, a relação do IIS com os eventos que o produzem configura uma macrocontingência. Entretanto, se ampliarmos a análise e considerarmos esse índice como um desdobramento das medidas estabelecidas por CCEs do governo estadual, podemos também interpretá-lo como um PA sem efeito selecionador, mas que influencia a consequência cultural, ou seja, a taxa de contágio (número de pessoas para as quais um infectado transmite o vírus). Independentemente de ser observado como produto de uma metacontingência ou efeito de uma macrocontingência, o IIS é um evento essencial por constituir um indicador dos comportamentos de toda sociedade que se pode mensurar e sobre o qual se pode intervir para, então, afetar o evento selecionador final e mantenedor de toda esta cadeia, a taxa de contágio por coronavírus. É essa taxa que se pretende achatar, afinal, ela tem implicações práticas sobre o sistema de saúde e sinaliza a conversão em óbitos de uma parcela das pessoas contaminadas. Por esses motivos, alterações da taxa de contágio têm o potencial de retroagir sobre as contingências entrelaçadas que produzem as prescrições governamentais.

A análise conjunta de todos os eventos leva a considerações relevantes. A intervenção realizada pelo Governo de São Paulo ilustra um fenômeno já descrito em outros estudos interpretativos, isto é, mudanças de comportamentos em grande escala por parte da população (entrelaçados ou não) dependem da promoção de metacontingências por parte do governo e outras instituições (e.g., Bortoloti \& D'Agostino, 2007; Fava, 2014). Um outro aspecto se refere ao encadeamento de eventos de tamanha complexidade. Cabral e Todorov 
(2015), por exemplo, já tinham demonstrado, em uma análise interpretativa, o encadeamento de PAs e a existência de uma metacontingência abrangendo outras metacontingências. No presente trabalho, além das metacontingências que ocorrem na esfera governamental, notamos o encadeamento de PAs com macrocomportamentos e a existência de macrocontingências dentro da metacontingência principal.

Se considerarmos, ainda, que a Figura 1 representa o encadeamento de eventos dinâmicos e recorrentes, outras pontuações precisam ser feitas. Primeiro, IIS e taxa de contágio estão presentes, em algum nível, o tempo inteiro. Não são efeitos da intervenção acrescidos ou retirados pontualmente do ambiente. Dessa forma, suas alterações é que são os indicadores da intervenção. Tais alterações, contudo, estão sujeitas aos mecanismos de ação da COVID-19 (e.g., aparecimento dos sintomas muitos dias após o contágio e pessoas assintomáticas), o que faz com que as mudanças produzidas demorem a retroagir sobre os culturantes que as produziram. Idealmente, a execução das medidas planejadas pelo governo resultaria em um alto IIS e em uma baixa taxa de contágio, o que implicaria em achatamento da curva de contágio pelo coronavírus. Entretanto, desde o início da adoção das medidas prescritas, esses indicadores oscilaram. O IIS em São Paulo se manteve sempre abaixo dos $70 \%$ recomendados (Governo de SP, 2020a) e a taxa de contágio do último mês sugere que uma medida mais drástica, como o lockdown, pode ser necessária (Pedrosa, 2020).

Não é objetivo do presente trabalho avaliar os efeitos das medidas adotadas. Porém, nossas análises permitem inferências sobre a recorrência de alguns desses eventos ao longo da pandemia. As datas indicadas para a adoção das medidas (apresentadas entre parênteses nos retângulos correspondentes aos PAs) mostram que novas prescrições foram feitas e algumas modificadas ao longo do tempo, possivelmente, indicando efeitos de retroação da consequência cultural em momentos distintos. Como se trata de uma cadeia longa de eventos, envolvendo metacontingência e macrocontingência, consideramos que uma mesma consequência cultural tenha efeitos opostos sobre CCEs e macrocomportamentos.

A diminuição da taxa de contágio poderia funcionar como contexto para a manutenção e prorrogação das medidas adotadas e, simultaneamente, ser entendida como indício de que a situação não é tão grave, levando à diminuição da probabilidade de macrocomportamentos. Isto implicaria em retomada de crescimento da taxa de contágio. A alta taxa de contágio, por outro lado, deve promover variação nas CCEs, gerando alterações nas prescrições prévias e proposição de novas medidas (e.g., interdição de estabelecimentos comerciais) capazes de reduzi-la. Podemos inferir também, que os efeitos das medidas adotadas acabam se sobrepondo ao longo do tempo, se convertendo em regras sobre as estratégias adotadas que ganham algum controle sobre as recorrências dos comportamentos Paradoxalmente, quando intervenções em saúde pública produzem bons efeitos, regras sobre o caráter alarmista e excessivo da intervenção podem surgir.

\section{PLANEJAMENTO CULTURAL NAS INTERVENÇÕES DO GOVERNO DO ESTADO DE SÃO PAULO}

Intervenções culturais seguem a mesma lógica das do nível operante: modificação de contingências (antecedentes e consequentes) para que haja variação, seleção ou extinção de respostas-alvo. Skinner (1961) sugere o planejamento cultural como estratégia de intervenção no nível cultural. Descrevemos planejamento cultural como: 1) modificação planejada de condições antecedentes que sirvam de contexto para 2) ocorrência de práticas culturais 3) selecionadas por consequências em curto prazo de modo que 4), em longo prazo, resultam na sobrevivência dessa cultura (Valderlon \& Elias, 2019). Práticas culturais são conjuntos de comportamentos individuais realizados de modo semelhante, transmitidos através de gerações (Skinner, 1953) e, portanto, podem ser compostas por comportamentos sociais, macrocomportamentos e culturantes. A principal diferença da intervenção nesses dois níveis é que a intervenção cultural visa modificação de comportamentos de várias pessoas de modo concomitante e os resultados dessas modificações devem ter efeitos mantenedores no presente ao mesmo tempo em que produzam resultados no futuro, como condições que permitam a sobrevivência das próximas gerações (Melo, Castro, \& de Rose, 2015). A atual pandemia consiste em um problema social extremamente relevante e, portanto, as intervenções culturais atuais com foco em seleção de práticas culturais que favoreçam o seu combate podem ser discutidas como estratégias de planejamento cultural.

Como modificação de condições antecedentes para aumento do IIS, uma estratégia utilizada pelo Governo de São Paulo foi a realização de campanhas de conscientização (notícias, aviso, propagandas) provavelmente, pelo baixo custo de resposta e capacidade de alcançar muitas pessoas ao mesmo tempo (Valderlon \& Elias, 2019). Essas campanhas podem servir como regras que descrevem as consequências reforçadoras para respostas de isolamento social e punitivas para respostas de descumprimento. Contudo, contingências verbais (e.g., campanhas de conscientização) podem não controlar comportamento, haja vista que em termos de probabilidade, contingências físicas (e.g., fechamento de escolas e comércios) podem controlar respostas de maneira mais eficiente (Lé Sénéchal-Machado \& Todorov, 2008). Como modificação de condições antecedentes no ambiente físico citamos o fechamento de escolas e serviços não essenciais, contexto para o macrocomportamento de sair de casa, diminuindo a probabilidade desse macrocomportamento ocorrer.

$\mathrm{Na}$ prática, o isolamento social implica em um contexto de privação, sobretudo, de recursos financeiros e de subsistência para a maior parte da população. Essas condições funcionam como operações motivadoras para 
respostas opostas ao isolamento social, ou seja, quem não tem recursos tende sair de casa para conseguir o sustento. Uma estratégia que funcionaria como arranjo de consequências em curto prazo, seria garantir condições mínimas de subsistência à população. Com esse objetivo, o governo federal instituiu a Lei $\mathrm{N}^{\circ} 13.982$ referente ao auxílio emergencial mensal de $\mathrm{R} \$ 600,00$ para trabalhadores informais, contribuintes informais, microempreendedores individuais e desempregados (Fortes, Schramm, Rego, Brito, \& Narciso, 2020). Esse auxílio pode ser entendido como contingência de suporte (Tadaiesky \& Tourinho, 2012), visto que são consequências individuais fornecidas pela agência de controle governamental, que selecionam respostas de entrelaçamentos, relacionados ao isolamento social. Contudo, a implementação dessa estratégia acabou gerando o efeito oposto, pois, no dia do pagamento do primeiro lote, muitas pessoas tiveram que ir às agências bancárias, provocando aglomerações e não havendo diminuição significativa do índice $(48 \%$ no estado de São Paulo no dia 27 de abril de 2020). O Governo de São Paulo também incentivou a economia com créditos subsidiários para empresas e suspensão de dívidas para empresas e pessoas físicas (Governo de São Paulo 2020b). Tais estratégias podem diminuir o efeito das operações motivadoras que dificultam o isolamento social.

Outra estratégia com arranjo de consequências em curto prazo para a seleção de respostas de isolamento social seria o uso de controle aversivo. Algumas intervenções culturais em situações experimentais têm se mostrado eficientes com uso desse tipo de controle do comportamento (e.g., Alves, Carvalho Neto, \& Tourinho, 2018; Critchfield, 2014; Guimarães, Picanço, \& Tourinho, 2019). O Governo de São Paulo começou a fazer sanções sociais como avisos e reprimendas por agentes públicos a pessoas nas ruas ou em aglomeração, funcionando como punição positiva para essas respostas. Além disso, pessoas sem máscaras na rua poderiam ser multadas, funcionando como punição positiva, ou impedidas de acessar lugares e transporte público, funcionando como reforçamento negativo para respostas de uso de máscaras.

Nesse sentido, empresas de produtos e serviços não essenciais foram proibidas de continuar as atividades e empresas de serviços essenciais deveriam seguir regras de controle de quantidade de pessoas e disponibilização obrigatória de produtos de limpeza individual. O descumprimento dessas regras implicaria em sanções, como avisos e notificações, e multas entre R \$ 276,00 a R\$ 276 mil dependendo do caso (Exame, 2020), ou seja, arranjando consequências culturais punidoras positivas contingentes a culturantes que desfavorecem o isolamento social (Governo de São Paulo 2020b).

Segundo a lógica do planejamento cultural, o resultado em longo prazo das intervenções realizadas pelo Governo de São Paulo deve ser a sobrevivência da cultura, ou seja, a garantia de condições de vida para as próximas gerações (Melo et al., 2015). Há ainda outros resultados relacionados à qualidade de vida das gerações futuras como um sistema de saúde que deve ficar mais estruturado para acolher as demandas de urgência e emergência pós- pandemia, vacinas e tratamentos para a COVID-19, planos de contingência mais eficientes para controle de pandemias e fortalecimento da ciência como área confiável de produção de conhecimentos e tecnologias. Todos esses resultados em longo prazo devem ser levados em consideração numa intervenção cultural e, analisando o presente, há razões para imaginar que esses resultados podem ocorrer.

\section{CONCLUSÃO}

O achatamento da taxa de contágios é fundamental para a preservação de vidas, além da evitação do colapso do sistema de saúde nacional (Ferguson et al., 2020). Para isso, Anderson et al. (2020) apontam que intervenções comportamentais são cruciais para controlar as taxas de contágio da COVID-19. Partindo desse ponto, intervenções sobre as respostas operantes, apenas, parecem ser suficientes para aumentar o índice de isolamento social. Contudo, o presente trabalho, que teve como objetivos: descrever metacontingências e macrocontingências envolvidas em medidas adotadas pelo Governo do Estado de São Paulo para promoção do isolamento social; e discutir tais medidas à luz do planejamento cultural, demonstrou que as práticas culturais implicadas nessas intervenções são complexas, uma vez que há arranjos tanto de metacontingências, quanto de macrocontingências.

Assim, os resultados apresentados neste trabalho corroboram a utilidade de metacontingência e macrocontingência como unidades de análise na interpretação de intervenções culturais (e.g., Bortoloti \& D’Agostino, 2007; Fava, 2014, Lé Sénéchal-Machado \& Todorov, 2008). Somada a esse tipo de produção, a peculiaridade dos achados (e.g., encadeamentos de eventos destacados na Figura 1) demonstra que fenômenos culturais ocorridos na complexidade dos ambientes naturais podem se configurar de múltiplas maneiras, o que ratifica a necessidade de estudos interpretativos usando tais ferramentas conceituais. Contudo, os questionamentos de Zilio (2019) sobre a efetividade da metacontingência na promoção de ações efetivas que solucionem problemas humanos são pertinentes. Mesmo que tenha identificado metacontingências, o presente trabalho apenas analisa uma intervenção feita por terceiros. Não se trata da aplicação de uma intervenção delineada a partir do conhecimento sobre metacontingências. Entretanto, isso não indica que não poderia sê-lo. Não obstante as dificuldades de um analista do comportamento de se colocar em uma posição que tenha poder de planejar e aplicar intervenções culturais, Lemos (2018) já demonstrou que é possível fazê-lo. Dado o atual caráter primordial das estratégias não farmacêuticas, que consistem basicamente em mudanças de comportamento em larga escala, a participação de analistas do comportamento em tais deliberações seria bastante oportuna.

Diante das análises do presente trabalho, nos parece que apenas as consequências individuais reforçadoras (e.g., auxílio emergencial) não seriam suficientes para a seleção e manutenção dos comportamentos de isolamento social, por isso outras 
medidas, mesmo sob controle aversivo, como descrito acima, deveriam ser implementadas para, em curto prazo, ajudar na seleção e manutenção desses comportamentosalvos. Embora essas operações de controle aversivo sejam usualmente aplicadas no cotidiano e, geralmente, identificadas pela Análise do Comportamento como maléficas para o indivíduo e para a cultura (Sidman, 1989/2003), elas nos encaminham para questionamento de ordem ética. Se ética implica na seleção de respostas operantes para o bem da cultura, ao falarmos do nível de seleção cultural (Dittrich \& Abib, 2004), seria então justificável usar controle aversivo para promover respostas de isolamento social durante a pandemia? Segundo Dittrich e Abib (2004), o discurso ético de analistas do comportamento pode ser generalizável e flexível, o que possibilita, a depender da situação, o uso de tais operações para aumentar as chances de sobrevivência da cultura. Nesse sentido, a promoção de medidas para salvar vidas, seja por reforçamento positivo e/ou controle aversivo, parecem ser imprescindíveis. Ainda assim, o uso de controle aversivo deveria ser pensado com cautela e baseado em achados empíricos, os quais têm demonstrado, apesar de incipientes, auxiliar na seleção e manutenção de classes de operantes e culturantes sob condições de controle aversivo envolvendo metacontingências (e.g., Alves et al., 2018; Guimarães et al., 2019).

Por fim, destacamos algumas das limitações metodológicas deste estudo. Com relação aos dados analisados, apenas estratégias listadas pelo site do governo foram consideradas, o que pode não refletir a totalidade ou o rigor das medidas adotadas. Sobre o escopo da análise, sabemos que as milhares de cidades brasileiras apresentam características geoeconômicas e culturais diversas e têm o poder de definir e implantar medidas próprias de combate à pandemia. Estudos futuros podem abarcar decisões estaduais e federais, mas devem considerar a possibilidade de adoção do recorte municipal para a produção de análises mais precisas. Quanto à esfera estadual, lidamos apenas com o conjunto de medidas denominadas pelo governo de São Paulo como promotora de Restrição de Circulação. Os outros 8 conjuntos de medidas muito provavelmente envolvem manipulação de variáveis que afetam o isolamento social, como a ampliação do registro de crimes pela Delegacia Eletrônica (Medida Administrativa) ou os acordos para a suspensão de corte de luz e gás durante a pandemia (Medida Social). No âmbito federal, outras medidas além do auxílio emergencial poderiam ser exploradas. Como demonstraram Ajzenman et al. (2020), as declarações do presidente do país influenciam o IIS. As disparidades entre as declarações dele e dos ministros da Saúde escolhidos por ele (que já somam três em pouco mais de dois meses de pandemia) tornam as variáveis promotoras de isolamento social ainda mais intrincadas e deveriam ser consideradas tanto na análise, quanto em uma possível intervenção.

\section{DECLARAÇÃO DE CONFLITOS DE INTERESSES}

Os (as) autores (as) declaram que não há conflito de interesses relativos à publicação deste artigo.

\section{CONTRIBUIÇÃO DE CADA AUTOR}

Certificamos que todos os autores participaram suficientemente do trabalho para tornar pública sua responsabilidade pelo conteúdo. A contribuição de cada autor pode ser atribuída como se segue: V. C. Amorim; T. M. M. Guimarães; J. A. T. Almeida; Y. Valderlon; M. Abdala; contribuíram para a concepção do artigo, pesquisa e redação do manuscrito.

\section{DIREITOS AUTORAIS}

Este é um artigo aberto e pode ser reproduzido livremente, distribuído, transmitido ou modificado, por qualquer pessoa desde que usado sem fins comerciais. $\mathrm{O}$ trabalho é disponibilizado sob a licença Creative Commons 4.0 BY-NC.

\section{(cc) BY-NC}

\section{REFERÊNCIAS}

Agbota, T., Sandaker, I., de Carvalho, L., \& Couto, K. (2017). Behavioral and cultural accounts of corruption in the interface between public officer and client. Revista Brasileira de Análise do Comportamento, 13(1), 29-41. doi: 10.18542/rebac.v13i1.5261

Ajzenman, N., Cavalcanti, T., \& Da Mata, D. (2020). More than Words: Leaders' Speech and Risky Behavior During a Pandemic. Social Science Research Network. doi: 10.2139/ssrn.3582908

Alves, L. F. C., Carvalho Neto, M. B., \& Tourinho, E. Z. (2018). Efeitos de consequências culturais análogas a reforçamento negativo sobre a recorrência de culturantes em microculturas de laboratório. Acta Comportamentalia, 26(2), 217-231.

Anderson, R. M., Heesterbeek, H., Klinkenberg, D., \& Hollingsworth, T. D. (2020). How will country-based mitigation measures influence the course of the COVID-19 epidemic? The Lancet, 395(10228), 931 934. doi: $10.25561 / 77735$

Ardila Sánchez, J. G., Houmanfar, R. A., \& Alavosius, M. P. (2019) A Descriptive Analysis of the Effects of Weather Disasters on Community Resilience. Behavior and Social Issues, 28, 298-315. doi: $10.1007 / \mathrm{s} 42822-019-00015-\mathrm{w}$

Azevedo, A. L. (2020, 10 de abril). Coronavírus: Brasil é o país que menos testa entre mais atingidos pela Covid-19. Recuperado de https://oglobo.globo.com/sociedade/coronavirus/coro navirus-brasil-o-pais-que-menos-testa-entre-maisatingidos-pela-covid-19-24363482

Bortoloti, R., \& D’Agostino, R. G. (2007). Ações pelo controle reprodutivo e posse responsável de animais domésticos interpretados à luz do conceito de metacontingência. Revista Brasileira de Análise do Comportamento, 3(1), 17-28. doi: 10.18542/rebac.v3i1.821.

Cabral, M., \& Todorov, J. (2015). Contingência e metacontingências no processo legislativo da lei sobre a remição da pena pelo estudo. Revista Brasileira de 
Análise do Comportamento, 11(2), 195-202. doi: 10.18542/rebac.v11i2.4013

Cidade de São Paulo. (2020). Coronavírus. Recuperado de

https://www.prefeitura.sp.gov.br/cidade/secretarias/sa ude/vigilancia_em_saude/doencas_e_agravos/coronavi rus/index.php?p=291766

Correio Braziliense (2020, 26 de março). Governadores desafiam o presidente e mantêm regras de isolamento. Recuperado de https://www.correiobraziliense.com.br/app/noticia/politi ca/2020/03/26/interna_politica,836890/governadoresdesafiam-o-presidente-e-mantem-regras-deisolamento.shtml

Critchfield, T. S. (2014). Skeptic's corner: Punishment Destructive force or valuable social "adhesive"? Behavior Analysis Practice, 7, 36-44. doi: 10.1007/s40617-014-0005-4

da Hora, K. L., \& Sampaio, A. A. S. (2019). Units of Analysis for Corruption Experiments: Operant, Culturobehavioral Lineage, Culturant, and Macrobehavior. Perspectives on Behavior Science, 42, 751-771. doi: 10.1007/s40614-019-00225-y

Dittrich, A., \& Abib, J. A. D. (2004). O sistema ético skinneriano e conseqüências para a prática dos analistas do comportamento. Psicologia: Reflexão e Crítica, 17(3), 427-433. doi: 10.1590/S010279722004000300014

Ellis, J., \& Magee, S. (2007). Contingencies, macrocontingencies, and metacontingencies in current educational practices: no child left behind? Behavior and Social Issues, 16, 5-26. doi: 10.5210/bsi.v16i1.361

Exame (2020, 5 de maio). Não usar máscara em SP pode levar a multa de até $\mathrm{R} \$ 276$ mil e detenção. Recuperado de https://exame.abril.com.br/brasil/decreto-demascaras-preve-multa-de-ate-r-276-mil-e-detencao-emsao-paulo/

Fava, V. M. D. (2014). Comportamento das famílias beneficiárias do Programa Bolsa Família: uma perspectiva analítico-comportamental do cumprimento das condicionalidades de educação e de saúde. (Tese de Doutorado). Universidade de Brasília, Brasília. Recuperado https://repositorio.unb.br/handle/10482/17831

Ferguson, N. M., Laydon, D., Nedjati-Gilani, G., Imai, N., Ainslie, K., Baguelin, M., ... Ghani, A. C. (2020). Impact of non-pharmaceutical interventions (NPIs) to reduce COVID-19 mortality and healthcare demand. (Relatório No. 9). Recuperado do site do Imperial College London. doi: 10.25561/77482

Fortes, P. D., Schramm, F. R., Rego, S., Brito, L., \& Narciso, L. (2020). O direito à renda básica no Brasil em tempos de Covid-19. Repositório Institucional da Fiocruz. Recuperado de https://www.arca.fiocruz.br/handle/icict/40786

Glenn, S. S. (2003). Operant contingencies and the origin of cultures. In K. A. Lattal \& P. N. Chase (Eds.). Behavior theory and philosophy (pp. 223-242). New York, NY: Kluwer Academic/Plenum Publishers. doi: 10.1007/9781-4757-4590-0_12
Glenn, S. S., Malott, M. E., Andery, M. A. P. A., Benvenuti, M., Houmanfar, R. A., Sandaker, I., ... Vasconcelos, L. A. (2016). Toward consistent terminology in a Behaviorist approach to cultural analysis. Behavior and Social Issues, 25, 11-27. doi: 10.5210/bsi.v25i0.6634

Governo de São Paulo (2020a). Sistema de Monitoramento Inteligente do Governo de São Paulo atualiza diariamente índice de adesão ao isolamento social no Estado. Recuperado de https://www.saopaulo.sp.gov.br/coronavirus/isolamento

Governo de São Paulo (2020b). Saiba quais as medidas do Governo de SP para o combate ao coronavírus. Recuperado de https://www.saopaulo.sp.gov.br/spnoticias/saiba-quaisas-medidas-do-governo-de-sp-para-o-combate-aocoronavirus-2/

Governo de São Paulo (2020c). Governo de SP apresenta Sistema de Monitoramento Inteligente contra coronavírus. Recuperado de https://www.saopaulo.sp.gov.br/noticiascoronavirus/governo-de-sp-apresenta-sistema-demonitoramento-inteligente-contra-coronavirus/

Governo de São Paulo. (2020d). Governo do Estado adota orientação educativa em quarentena. Recuperado de https://www.saopaulo.sp.gov.br/noticiascoronavirus/governo-do-estado-adota-orientacaoeducativa-em-quarentena/

Governo de São Paulo (2020e). Governo de SP determina uso obrigatório de máscaras em todo o estado. Recuperado de https://www.saopaulo.sp.gov.br/noticiascoronavirus/governo-de-sp-determina-uso-obrigatoriode-mascaras-em-todo-o-estado/

Guimarães, T. M. M., Picanço, C. R. F., \& Tourinho, E. Z. (2019). Effects of Negative Punishment on Culturants in a Situation of Concurrence between Operant Contingencies and Metacontingencies. Perspectives on Behavior Science, 42(4), 733-750. doi: 10.1007/s40614 019-00224-z

Kissler, S. M., Tedijanto, C., Goldstein, E., Grad, Y. H., \& Lipsitch, M. (2020). Projecting the transmission dynamics of SARS-CoV-2 through the postpandemic period. Science, 368(6493), 860-868. doi: 10.1126/science.abb5793

Lemos, R. F. (2018). A atuação do analista do comportamento em políticas públicas: ação intersetorial em âmbito local para aumentar a frequência escolar de adolescentes de famílias beneficiárias do Programa Bolsa Família. (Tese de Doutorado). Universidade de Brasília, Brasília. Recuperado de https://repositorio.unb.br/handle/10482/32800.

Lé Sénéchal-Machado, V., \& Todorov, J. C. (2008). A Travessia na faixa de pedestre em Brasília (DF/Brasil): exemplo de uma intervenção cultural. Revista Brasileira de Análise do Comportamento, 4(2), 191-204. doi: 10.18542/rebac.v4i2.850

Martins, A. L. (2009). O Sistema Único de Saúde: contingências e metacontingências nas leis orgânicas da saúde. (Dissertação de Mestrado). Universidade de Brasília, Brasília. Recuperado de https://repositorio.unb.br/handle/10482/7749 
Melo, C. M., Castro, M. S. L. B., \& de Rose, J. C. (2015). Some relations between culture, ethics, and technology in BF Skinner. Behavior and Social Issues, 24(1), 39-55. doi: 10.5210/bsi.v24i0.4796

Ministério da Saúde (2020). Painel coronavírus. Recuperado de https://covid.saude.gov.br/

OPAS/OMS Brasil (2020, 2 de junho). Folha informativa COVID-19 (doença causada pelo novo coronavírus). Recuperado de: https://www.paho.org/bra/index.php?option=com_conte nt $\&$ view $=$ article $\&$ id $=6101:$ covid $19 \&$ Itemid $=875$

Pedrosa, R. H. (2020). The dynamics of Covid-19: weather, demographics and infection timeline. medRxiv. doi: 10.1101/2020.04.21.20074450

Secretaria de Estado da Saúde (2020). Isolamento social em São Paulo é de $49 \%$, aponta Sistema de Monitoramento Inteligente. Recuperado de http://www.saude.sp.gov.br/ses/perfil/cidadao/homepage /destaques/isolamento-social-em-sao-paulo-e-de-49aponta-sistema-de-monitoramento-inteligente

Sidman, M. (2003). Coerção e suas implicações. São Paulo: Livro Pleno. (Originalmente publicado em 1989).

Singhal, T. (2020). A Review of Coronavirus Disease-2019 (COVID-19). The Indian Journal of Pediatrics, 87, 281286. doi: 10.1007/s12098-020-03263-6

Skinner, B. F. (1961). The design of cultures. Daedalus, 90(3), 534-546. Recuperado de http://www.jstor.org/stable/20026672

Skinner, B. F. (1953). Science and human behavior. New York: Macmillan.

Tadaiesky, L. T., \& Tourinho, E. Z. (2012). Effects of support consequences and cultural consequences on the selection of interlocking behavioral contingencies. Revista Latinoamericana de Psicologia, 44(1), 133-147.

Tagliabue, M., \& Sandaker, I. (2019). Societal Well-Being: Embedding Nudges in Sustainable Cultural Practices. Behavior and Social. Issues, 28, 99-113. doi: 10.1007/s42822-019-0002-X

Valderlon, Y., \& Elias, L. R. (2019). The Bolsa Família Program and cultural design: Behavioral interpretations of cultural interventions. Behavior and Social Issues, 28(1), 114-126. doi: 10.1007/s42822-019-0003-90003-9

Vieira, M. C., Andery, M. A. P. A., \& Pessôa, C. V. B. B. (2016). Condições antecedentes em metacontingências. Acta Comportamentalia, 24(4), 439-451.

Walker, P. G. T., Whittaker, C., Watson, O., Baguelin, M., Ainslie, K. E. C., Bhatia, S., ... Ghani, A. C. (2020). The Global Impact of COVID-19 and Strategies for Mitigation and Suppression. (Relatório No. 12). Recuperado do site do Imperial College London. doi: $10.25561 / 77735$

World Health Organization (2020). Coronavirus diesease (covid-2019) situation reports. Recuperado de https://www.who.int/docs/defaultsource/coronaviruse/situation-reports/20200622-covid19-sitrep-154.pdf?sfvrsn=d0249d8d_2

Zilio, D. (2019). On the Function of Science: an Overview of 30 Years of Publications on Metacontingency. Behavior and Social Issues, 28, 1-31. doi: 10.1007\%2Fs42822-019-00006-X
Submetido em: 18/05/2020 Aceito em: 25/05/2020 